\title{
CLOSURE THEORIES OF POWERSET THEORIES
}

\author{
JiŘÍ MOČKOř
}

\begin{abstract}
A notion of a closure theory of a powerset theory in a ground category is introduced as a generalization of a topology theory of a powerset theory. Using examples of powerset theories in the category Set of sets and in the category of sets with similarity relations, it is proved that these theories can be used as ground theories for closure theories of powerset theories in these two categories. Moreover, it is proved that all these closure theories of powerset theories are topological constructs. A notion of a closure operator which preserves a canonical form of fuzzy objects in these categories is introduced, and it is proved that a closure theory of a powerset theory in the ground category Set is a coreflective subcategory of the closure theory of (Zadeh's) powerset theory, which preserves canonical forms of fuzzy sets.
\end{abstract}

\section{Introduction}

In almost all branches of mathematics the notion of the powersets and powerset operators in classical set theory is one of the most useful and exploited tools. Recall that given a set $X$, there exists the set $\mathcal{P}(X)=\{S: Y \subseteq X\}$, called the powerset of $X$ and such that every map $f: X \rightarrow Y$ can be extended to the powerset operators $f^{\rightarrow}: \mathcal{P}(X) \rightarrow \mathcal{P}(Y)$ and $f^{\leftarrow}: \mathcal{P}(Y) \rightarrow \mathcal{P}(X)$, such that

$$
f^{\rightarrow}(S)=f(S), \quad f^{\leftarrow}(T)=f^{-1}(T) .
$$

The powerset structures are widely used in algebra, logic, topology and also in computer science, for illustrative examples of possible applications in see, e.g., the introductory part of the paper of [18], for applications of powerset objects in abstract interpretation see, e.g., [1], 6]. Because the classical set theory can be considered to be a special part of fuzzy set theory, introduced by [28, is natural that powerset objects associated with fuzzy sets soon were investigated as generalizations of classical powerset objects. A fuzzy set in a set $A$ with values

(C) 2015 Mathematical Institute, Slovak Academy of Sciences. 2010 Mathematics Subject Classification: 03E72.

Keywords: closure theory, powerset theory, set with a similarity relation, topological construct.

This work was supported by the Centre of Excellence project LQ1602. 


\section{JIŘÍ MOČKOř}

in some partly ordered structure $Q$ is defined as a map $A \rightarrow Q$ and an investigation of objects of all fuzzy sets $I^{X}$ was of interest. The first approach was done again by [28], who defined $I^{X}$ to be a new powerset object instead of $\mathcal{P}(X)$ and introduced new powerset operators $f_{Z}: I^{X} \rightarrow I^{Y}$ and $f_{Z}^{\leftarrow}: I^{Y} \rightarrow I^{X}$, such that for $s \in I^{X}, t \in I^{Y}, y \in Y$,

$$
f_{Z}(s)(y)=\bigvee_{x, f(x)=y} s(x), \quad f_{Z}^{\leftarrow}(t)=t \circ f .
$$

A lot of papers were published about Zadeh's extension and its generalizations, see, e.g., [22], 23], 25]. Zadeh's extension (which could be considered as an extension of a forward powerset operator $f \rightarrow$ ) was for the first time intensively studied by [14, especially the relation between $f \rightarrow$ and $f \vec{Z}$. This paper was in fact the first real attempt to uniquely derive the powerset operators $f_{Z}, f_{Z}$ from $f^{\rightarrow}$ and $f^{\leftarrow}$ and not only explicitly stipulate them. Whatever works of Rodabaugh gave very serious basis for further research of powerset objects and operators, only abstract theory of powerset objects based on similar principles as the theory of monads has brought another important ideas to the research of powerset objects. The principal idea of the categorical approach was an observation that classical powerset objects constitute the so-called algebraic theory (or monad), introduced by E. G. M a n es [19]. Roughly speaking, there exists an algebraic theory (in clone form) $\mathbf{P}=(\mathcal{P}, \eta, \diamond)$ in the category Set, where $\mathcal{P}(X)=2^{X}$ (see [2]), such that the operator $f_{\mathcal{P}}: \mathcal{P}(X) \rightarrow \mathcal{P}(Y)$ induced from $\mathbf{P}$ by setting $f_{\mathcal{P}}=\left(\eta_{B} \circ f\right) \diamond 1_{\mathcal{P}(X)}$ is the same as $f \rightarrow$. Similarly can be derived the powerset operator $f_{\mathcal{P}}^{\leftarrow}$. In this sense, an algebraic theory $\mathbf{P}$ generates the traditional powerset theory [2]. In the same manner as in the case of an algebraic theory $\mathbf{P}=(\mathcal{P}, \eta, \diamond)$, which generates powerset operators in the category Set, we can formally proceed in defining algebraic theory $\mathbf{Z}=(Z, \mu, \square)$ which defines powerset operators for $Q$-valued fuzzy sets, where $Q$ is an appropriate lattice. In that case, $Z(X)=Q^{X}$, and $\mu_{X}(x)$ is the $Q$-valued characteristic morphism of a subset $\{x\}$ in $X$ and such that for any $f: X \rightarrow Q^{Y}, g: Y \rightarrow Q^{Z}$, the clone composition $g \square f: X \rightarrow Q^{Z}$ is defined by

$$
[(g \square f)(x)](z)=\bigvee_{y \in Y} f(x)(y) \otimes g(y)(z) .
$$

S. E. R o d a b a u h [6] then proved that $\mathbf{Z}$ is an algebraic theory if and only if $(Q, \leq, \otimes)$ is a unital quantale with unit. Moreover, he also proved that in that case, for each morphism $f: X \rightarrow Y$, the lifting $\left(\mu_{X} \circ f\right) \square 1_{Z(X)}$ equals to the classical Zadeh's powerset operator $f_{Z}: Z(X) \rightarrow Z(Y)$. In that case, $Z$ : Set $\rightarrow$ Set is a functor, $Z(f)=f \vec{Z}$. Because a significant part of the theory of $Q$-fuzzy sets is built on some variants of residuated lattices which fulfill that condition, it is possible even in such cases to use algebraic theory for the construction of powerset operators. 


\section{CLOSURE THEORIES OF POWERSET THEORIES}

E. G. M a n es [20] introduced also a new structure $\mathbf{T}=\left(T, e,(-)^{*}\right)$, called fuzzy theory, such that $T$ assigns to each set $X$ a set $T(X)$, e assigns to each set $X$ a map $e_{X}: X \rightarrow T(X)$, and $(-)^{*}$ assigns to each function $f: X \rightarrow T(Y)$ a function $f^{*}: T(X) \rightarrow T(Y)$, satisfying some additional conditions. Fuzzy theory is, in fact, identical with the algebraic theory $(T, \diamond, e)$ in the category Set, if we set $g \diamond f=g^{*} \circ f$.

Instead of algebraic theory (in clone form) introduced by [19], more explicit powerset theory was introduced by S. E. R o d a b a u g h [16] as a special structure describing powerset objects. A slight modification of that structure defined in a category $\mathbf{K}$, is represented by a system $\mathbf{P}=(P, \rightarrow, V, \eta)$, where $P:|\mathbf{K}| \rightarrow C S L A T$ is a powerset generator (where CSLAT is the category of complete semilattices), $\rightarrow$ is a forward powerset operator, such that for each $f: X \rightarrow Y$ in $\mathbf{K}, f_{\mathbf{P}}: P(X) \rightarrow P(Y)$ in $C S L A T, V: \mathbf{K} \rightarrow$ Set is a concrete functor and $\eta_{X}: V(X) \rightarrow P(X)$ is a map for each object $X$. He then proved that some powerset theories can be generated by algebraic theories.

Since the original Z a d e h's paper was published, the notion of "fuzzy set" has been changed significantly and it is now more general. The first important modification concerns the value set: instead of real number interval $I=[0,1]$, more general lattice structures $Q$ are considered. Among these lattice structures, complete residuated lattices play important role, (see, e.g., 21]), in modern terminology unital and commutative quantale, (see 7]), i.e., a structure $Q=(L, \wedge, \vee, \otimes, \rightarrow, 0,1)$ such that $(L, \wedge, \vee)$ is a complete lattice, $(L, \otimes, 1)$ is a commutative monoid with operation $\otimes$ isotone in both arguments and $\rightarrow$ is a binary operation which is adjoint with respect to $\otimes$, i.e.,

$$
\alpha \otimes \beta \leq \gamma \quad \text { if and only if } \alpha \leq \beta \rightarrow \gamma .
$$

A well known example is the Eukasiewicz algebra $\mathrm{E}=\left([0,1], \vee, \wedge, \otimes, \rightarrow_{\mathrm{E}}, 0,1\right)$, where

$$
\begin{aligned}
a \otimes b & =0 \vee(a+b-1) \\
a \rightarrow_{\mathrm{E}} b & =1 \wedge(1-a+b) .
\end{aligned}
$$

Further, classical fuzzy sets (or even fuzzy sets with values in residuated lattice $Q$ ) were originally defined on sets. But any set $A$ can be considered as a couple $(A,=)$, where $=$ is a standard equality relation defined on $A$. It is then natural instead of the crisp equality relation $=$, to consider some more "fuzzy" equality relation defined on $A$, which is called a similarity relation. Hence, instead of a classical set $A$ as a basic set and a fuzzy set $s: A \rightarrow Q$, we can use a set with similarity relation $(A, \delta)$ (called a $Q$-set) and a map $s:(A, \delta) \rightarrow Q$. Such a map then represents some new "fuzzy object" in $(A, \delta)$. The next step in our generalization process is more abstract. Instead of maps $A \rightarrow Q$, or $(A, \delta) \rightarrow Q$, we can use more general structures, i.e., morphisms in some categories. In the fuzzy set theory various categories are used to describe 
properties of such "fuzzy objects". Properties of such categories were intensively investigated, e.g., 4. In the paper, we use a category with $Q$-sets as objects and naturly defined morphisms. A morphism $f:(A, \delta) \rightarrow(B, \gamma)$ in the category $\operatorname{Set}(Q)$ is a map $f: A \rightarrow B$ such that $\gamma(f(x), f(y)) \geq \delta(x, y)$ for all $x, y \in A$. It is then natural to speak about a fuzzy object $(A, \delta) \rightarrow(Q, \leftrightarrow)$ in the category $\operatorname{Set}(Q)$, instead of a "fuzzy set", where $\leftrightarrow$ is the biresiduation operation in $Q$ defined by $\alpha \leftrightarrow \beta=(\alpha \rightarrow \beta) \wedge(\beta \rightarrow \alpha)$. These fuzzy objects generalize classical fuzzy sets $A \rightarrow Q$. In fact, if we consider, for example, $Q$-sets $(A,=)$, then $s:(A,=) \rightarrow(Q, \leftrightarrow)$ is a morphism in $\operatorname{Set}(Q)$ if and only if $s: A \rightarrow Q$ is a map, i.e., a classical fuzzy set.

By using that general approach, we can even define fuzzy objects which are neither maps nor morphisms. The motivation for these fuzzy objects comes from $\alpha$-cuts of classical fuzzy sets. Any classically defined fuzzy set $X$ in a set $A$ with values in $Q$ can be defined equivalently by a system of level sets $X_{\alpha}, \alpha \in Q$, where $X_{\alpha}=\{a \in A: X(a) \geq \alpha\}$. Conversely, any (nested) system $\left(Y_{\alpha}\right)_{\alpha}$ of subsets of $A$ such that for any $a \in A$ the set $\left\{\alpha \in Q: a \in Y_{\alpha}\right\}$ has the greatest element (such system is called a cut in $A$ ), defines a fuzzy set $Y$ such that $Y(a)=\bigvee_{\left\{\beta: a \in Y_{\beta}\right\}} \beta$. In our previous papers [9], 10], we proved that analogously any morphisms $(A, \delta) \rightarrow(Q, \leftrightarrow)$ in the category $\operatorname{Set}(Q)$ can be defined equivalently by a system of some special subsets of $A$ or $A \times Q$, respectively, called f-cuts. Hence, f-cuts then represent another fuzzy objects in $(A, \delta)$ in our categories $\mathcal{K}$, which generalize classical fuzzy sets.

It is important to know that all these fuzzy objects represent object functions of functors from a corresponding category into the category Set (see [9, [10]), or even into the category CSLAT of all complete $\bigvee$-semilatices. It means, that sets of fuzzy objects can be ordered in a natural way, such that the resulting ordered sets are complete $\bigvee$-semilattices, and the above mentioned functors are, in fact, functors from corresponding categories to the category CSLAT.

For these new fuzzy objects it is important that the corresponding powerset objets, i.e., sets $F(A, \delta)$ of all morphisms $(A, \delta) \rightarrow(Q, \leftrightarrow)$ and sets $C(A, \delta)$ of all f-cuts have analogical properties to those of classical fuzzy sets. In the paper [12] we proved that all these fuzzy objects has powerset structures which are powerset theories in corresponding categories, in the sense of Rodabaugh and some of these powerset theories are defined by algebraic theories. For classical powerset theories $\mathbf{Z}$ and $\mathbf{P}$ there exists a strong relation between these two theories, which can be represented as some homomorphism $\mathbf{P} \rightarrow \mathbf{Z}$. In [12] we proved that analogously for these new fuzzy theories $\mathbf{F}$ there exist "new classical" powerset theories $\mathbf{R}$ and a homomorphism $\mathbf{R} \rightarrow \mathbf{F}$.

Algebraic theories and powerset theories were used to develop a fuzzy topological theories for lattice-valued mathematics. The basic idea of this approach is the definition of a new theory that contains, among powerset objects also 


\section{CLOSURE THEORIES OF POWERSET THEORIES}

topology defined on these powerset objects, and the inclusion of relevant axioms concerning continuity to this new theory (see, e.g., S. E. Rodaba ugh [16], S. A. Solovyov [18, with extended list of references).

In this paper we want to investigate powerset objects in ground categories Set and $\operatorname{Set}(Q)$ in two different but interconnected sections. Firstly, we introduce the notion of a closure theory of a powerset theory in a ground category as a natural generalization of a topology theory of a powerset theory, which was previously defined by S. E. R o d a b a u g h [16. Using examples of powerset theories presented in previous papers of S. E. R o d a b a u g h [16], U. Höh le [13], J. M o čk or [12] and others, we show that these powerset theories can be used as ground theories for closure theories of powerset theories in a ground category Set or $\operatorname{Set}(Q)$ and that all these closure theories of powerset theories are topological constructs, which also generalizes results of S. E. R o d a b a u g h [16].

The other section will deal with properties of objects of closure theories of powerset theories. In our previous paper [11] we proved several extension theorems for closure operators, under which a closure operator defined on a powerset structure of one type can be extended to a closure operator defined on another powerset structure. We also investigated relationships between continuity of pairs of morphisms $f, g$ in categories of powerset structures, with respect to a closure operators, where $f$ and $g$ are morphisms between powerset structures of different types. In the paper we will continue in such investigation of these properties. An important notion will be the notion of a closure operator which preserves a canonical form of fuzzy objects. Using that notion we will describe some extensions of closure operators. Moreover, using that notion we show that a closure theory of a classical powerset theory in the ground category Set is a coreflective subcategory in the subcategory of closure theory of (Zadeh's) powerset theory, which is based on closure operators which preserves a canonical forms of (Zadeh's) fuzzy sets.

\section{Preliminaries}

For a convenience of potential readers we repeat in this section several notions and facts about sets with similarity relations and the category $\operatorname{Set}(Q)$ of these sets. We also repeat some results about special morphisms in the category $\operatorname{Set}(Q)$, which are considered to be a generalization of classical fuzzy sets. Some results will be also mentioned about isomorphisms between these fuzzy sets and special cut systems in the category $\operatorname{Set}(Q)$, which generalize relationships between classical fuzzy sets and $\alpha$-cuts. We also repeat some basic theorem about extensions of closure operators, under which a closure operator defined on one powerset structure can be extended to a closure operator defined on another powerset structure. Most of results were published in the papers [9]-[11]. 


\section{JIŘí MOČKOŘ}

In the paper the symbol $Q$ is used for a a structure $Q=(L, \wedge, \vee, \otimes, \rightarrow, 0,1)$ such that $(L, \wedge, \vee)$ is a complete lattice, $(L, \otimes, 1)$ is a commutative monoid with operation $\otimes$ isotone in both arguments and $\rightarrow$ is a binary operation which is adjoint with respect to $\otimes$, i.e.,

$$
\alpha \otimes \beta \leq \gamma \quad \text { if and only if } \quad \alpha \leq \beta \rightarrow \gamma
$$

Recall that a system $\left(C_{\alpha}\right)_{\alpha \in Q}$ is a cut systems in a set $X$, if $C_{\alpha} \subseteq X$ and if $a \in X$ is such that $\bigvee_{\beta: a \in C_{\beta}} \beta \geq \alpha$, then $a \in C_{\alpha}$. It is well known that there exists a bijection between the set of all cut systems in a set $X$ and a set $Q^{X}$.

Recall that a set with similarity relation (or $Q$-set) is a couple $(A, \delta)$, where $\delta: A \times A \rightarrow Q$ is a map such that

(a) $(\forall x \in A) \delta(x, x)=1_{Q}$,

(b) $(\forall x, y \in A) \delta(x, y)=\delta(y, x)$,

(c) $(\forall x, y, z \in A) \delta(x, y) \otimes \delta(y, z) \leq \delta(x, z)$ (generalized transitivity).

We will use the category $\operatorname{Set}(Q)$ with $Q$-sets as objects and with morphisms $f:(A, \delta) \rightarrow(B, \gamma)$ defined as a map $f: A \rightarrow B$, such that $\gamma(f(x), f(y)) \geq \delta(x, y)$, for all $x, y \in A$. The category $\operatorname{Set}(Q)$ has its origin in Wyler's category introduced in [27, and in a more general way developed by U. Hö h le in [4. The category $\operatorname{Set}(Q)$ and some its modifications are frequently used as a natural background for categorical investigation of fuzzy set theory.

Cut systems (called $f$-cut systems) can be defined also in the category $\operatorname{Set}(Q)$ (see [9], 10]), where a system of subsets $\left(C_{\alpha}\right)_{\alpha \in Q}$ of $A$ is an f-cut system in a $Q$-set $(A, \delta)$, if

(a) $\forall a, b \in A, \quad a \in C_{\alpha} \Rightarrow b \in C_{\alpha \otimes \delta(a, b)}$,

(b) $\forall a \in A, \forall \alpha \in Q, \bigvee_{\left\{\beta: a \in C_{\beta}\right\}} \beta \geq \alpha \Rightarrow a \in C_{\alpha}$.

We will use the following notations for powerset objects:

- $P(A)=\left(2^{A}, \subseteq\right), A \in|\operatorname{Set}|$,

- $Z(A)=\left(Q^{A}, \leq\right)$, ordered pointwise, $A \in \mid$ Set $\mid$,

- $D(A)$ is the set of all cut systems in $A$, ordered by inclusion, $A \in \mid$ Set $\mid$,

- $F(A, \delta)=H_{\text {om }} \operatorname{Set}(Q)((A, \delta),(Q, \leftrightarrow))$, ordered pointwise, $(A, \delta) \in|\operatorname{Set}(Q)|$,

- $C(A, \delta)$ is the set of all f-cut systems in $(A, \delta)$ in the category $\operatorname{Set}(Q)$, ordered by inclusion, $(A, \delta) \in|\operatorname{Set}(Q)|$, and

- $R(A, \delta)=\left(2^{A}, \subseteq\right),(A, \delta) \in|\operatorname{Set}(Q)|$.

In the paper [8] we proved that for every $Q$-set $(A, \delta)$, there exists a map : $Z(A) \rightarrow F(A, \delta)$ defined by

$$
\widehat{s}(a)=\bigvee_{x \in A} s(x) \otimes \delta(a, x),
$$




\section{CLOSURE THEORIES OF POWERSET THEORIES}

and this map is a closure operator on the set $Z(A)$. Analogously, in the paper [9] we proved that there exists a map ${ }^{\sim}: D(A) \rightarrow C(A, \delta)$, defined by

$$
\widetilde{\left(C_{\alpha}\right)_{\alpha}}=\left(\widetilde{C_{\alpha}}\right)_{\alpha}, \quad \widetilde{C_{\alpha}}=\left\{a \in \underset{\left\{(x, \beta): x \in C_{\beta}\right\}}{\left.A: \bigvee_{\beta} \beta \delta(a, x) \geq \alpha\right\} .}\right.
$$

We will use those closure operators and ${ }^{\sim}$ in the rest of the paper. In the paper [1] we investigated a possibility to extend a closure operator defined on a powerset objects of some fuzzy objects to a closure operator defined on another powerset object of fuzzy objects. For example, if $c$ is a closure operator defined on an ordered set $(Z(A), \leq)$ of fuzzy sets in a set $A$, then using $c$, another closure operator $\psi_{Z(A), A}(c)$ can be defined on $A$. In fact, let $(A, \leq)$ be an ordered set and let $\mathcal{U}(A, \leq)$ be the set of all closure operators defined on $(A, \leq)$. Then the following theorem was proved.

Theorem 2.1 ([11, Theorem 3.1]). Let $A$ be a set.

(1) There exist maps such that the diagram commutes.

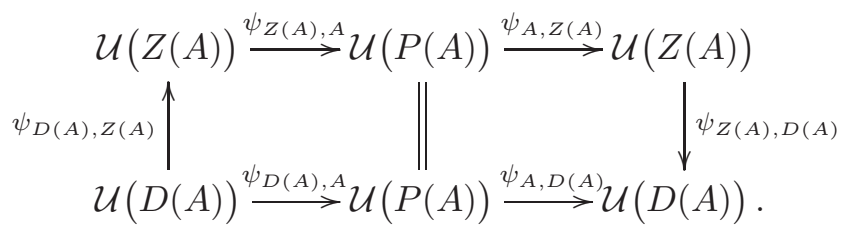

(2) $\psi_{Z(A), A} \circ \psi_{A, Z(A)}=i d_{\mathcal{U}(P(A))}$.

(3) $\psi_{D(A), A} \circ \psi_{A, D(A)}=i d_{\mathcal{U}(P(A))}$.

(4) $\psi_{D(A), Z(A)}$ and $\psi_{Z(A), D(A)}$ are mutually inverse maps.

Moreover, in [11] we also proved that it is possible to describe continuous properties of functors $P, Z, D$ with respect to closure operators. Recall that if $(V, c)$ and $(W, d)$ are ordered sets with closure operators, then a map $f: V \rightarrow W$ is continuous if $f(c(x)) \leq d(f(x))$, for any $x \in V$. The following theorem was proved (for the definition of $f_{P}, f_{Z}$ see examples in Section 3 ).

Proposition 2.1 ([11, Proposition 4.1]). Let $A, B$ be sets and let $c$ (d, respectively) be a closure operator on the set $A$ (B, respectively). Let $v=\psi_{A, Z(A)}(c)$, $w=\psi_{B, Z(B)}(d)$, and let $f: A \rightarrow B$ be a map. Then the following statements are equivalent.

(1) $f_{P}:(P(A), c) \rightarrow(P(B), d)$ is continuous.

(2) $f_{Z}:(Z(A), v) \rightarrow(Z(B), w)$ is continuous.

Analogical results can be obtained for closure operators defined on object functions of functors $R, F, C$. In fact the following theorem and proposition was proved, where $\tilde{\mathcal{U}}(R(A, \delta))$ is the set of all closure operators $c$ defined on a po- 
werset $R(A, \delta)$, which satisfy the condition $c\left(\widetilde{E_{\alpha}}\right) \subseteq \widetilde{c\left(E_{\alpha}\right)}$, for every cut system $\left(E_{\alpha}\right)_{\alpha} \in D(A)$ and a given $Q$-set $(A, \delta)$.

Theorem 2.2 ([11, Theorem 3.2]). Let $(A, \delta)$ be a $Q$-set.

(1) There exist maps such that the diagram commutes.

$$
\begin{array}{ccc}
\mathcal{U}(F(A, \delta)) \stackrel{\varphi_{F(A, \delta), A}}{\longrightarrow} \mathcal{U}(R(A, \delta)) & \supseteq \widetilde{\mathcal{U}}(R(A, \delta)) \stackrel{\varphi_{A, F(A, \delta)}}{\longrightarrow} \mathcal{U}(F(A, \delta)) \\
\uparrow \varphi_{C(A, \delta), F(A, \delta)} & \| & \varphi_{F(A, \delta), C(A, \delta)} \downarrow \\
\mathcal{U}(C(A, \delta)) \stackrel{\varphi_{C(A, \delta), A}}{\longrightarrow} \mathcal{U}(R(A, \delta)) \supseteq \widetilde{\mathcal{U}}(R(A, \delta)) \stackrel{\varphi_{A, C(A, \delta)}}{\longrightarrow} \mathcal{U}(C(A, \delta)) .
\end{array}
$$

(2) $\varphi_{C(A, \delta), F(A, \delta)}$ and $\varphi_{F(A, \delta), C(A, \delta)}$ are mutually inverse maps.

(3) $\varphi_{F(A, \delta), A} \circ \varphi_{A, F(A, \delta)}=i d_{\widetilde{\mathcal{U}}(R(A, \delta))}$.

(4) $\varphi_{C(A, \delta), A} \circ \varphi_{A, C(A, \delta)}=i d_{\widetilde{\mathcal{U}}(R(A, \delta))} \cdot$

For the definition of $f_{R}, f_{F}$ in the next proposition see also examples in Section 3 .

Proposition 2.2 ([11, Proposition 4.3]). Let $(A, \delta),(B, \gamma)$ be Q-sets and let $c \in \widetilde{\mathcal{U}}(A)$ and $d \in \widetilde{\mathcal{U}}(B)$ be closure operators. Let $v=\varphi_{A, F(A, \delta)}(c), w=\varphi_{B, F(B, \gamma)}(d)$, and let $f:(A, \delta) \rightarrow(B, \gamma)$ be a morphism in the category Set $(Q)$. Let us consider the following statements:

(1) $f_{R}:(R(A, \delta), c) \rightarrow(R(B, \gamma), d)$ is continuous,

(2) $f_{F}:(F(A, \delta), v) \rightarrow(F(B, \gamma), w)$ is continuous.

Then $(1) \Rightarrow(2)$. If the closure operator $c_{B, \gamma}$ is trivial on the set $B$, then also $(2) \Rightarrow(1)$.

\section{Closure theory of a powerset theory}

Because of the convenience of the reader we repeat the basic definition of the powerset theory, which was introduced by S. E. R od ab u g h [16] as an analogy of algebraic theory in clone form.

Definition 3.1 (S. E. Rodabaugh [16]). Let $\mathbf{K}$ be a ground category. Then $\mathbf{P}=(P, \rightarrow, V, \eta)$ is called $C S L A T$-powerset theory in $\mathbf{K}$, if

(1) $P:|\mathbf{K}| \rightarrow|C S L A T|$ is an object-mapping,

(2) for each $f: A \rightarrow B$ in $\mathbf{K}$, there exists $f_{\mathbf{P}}: P(A) \rightarrow P(B)$ in CSLAT,

(3) for each $f: A \rightarrow B$ in $\mathbf{K}$, there exists $f_{\mathbf{P}}^{\leftarrow}: P(B) \rightarrow P(A)$ in $C S L A T$,

(4) $\left(f_{\mathbf{P}}, f_{\mathbf{P}}^{\leftarrow}\right)$ is a Galois connection, 


\section{CLOSURE THEORIES OF POWERSET THEORIES}

(5) there exists a concrete functor $V: \mathbf{K} \rightarrow$ Set, such that $\eta$ determines in Set for each $A \in \mathbf{K}$ a mapping $\eta_{A}: V(A) \rightarrow P(A)$,

(6) for each $f: A \rightarrow B$ in $\mathbf{K}, f_{\mathbf{P}} \circ \eta_{A}=\eta_{B} \circ V(f)$.

We will frequently deal with the following situation. Let $\mathbf{K}$ be a category and let $P: \mathbf{K} \rightarrow C S L A T$ be a covariant functor. It follows that for any morphism $f: A \rightarrow B, P(f)$ is a map preserving all sup. Instead of $P(f)$, we use $f_{P}$.

By using the well known Adjoint Functor Theorem (see, e.g., [5], [15]) for any morphism $f: A \rightarrow B$ in $\mathbf{K}$, there exists the map $f_{P}^{\leftarrow}: P(B) \rightarrow P(A)$ defined by

$$
\begin{aligned}
(\forall Y \in P(B)) \quad f_{P}^{\leftarrow}(Y)= & \bigvee X . \\
\left\{X \in P(A): f_{P}(X) \leq Y\right\} &
\end{aligned}
$$

It is then clear that $f_{P}^{\overleftarrow{P}}: P(B) \rightarrow P(A)$ preserves all existing meets and $\left(f_{P}, f_{P}^{\overleftarrow{P}}\right)$ is a Galois connection. If a functor $P$ will be given, then by $f_{P}^{\leftarrow}$ we will understand the map defined by (11) from $P(f)=f_{P}$.

In the paper we will deal with several examples of $C S L A T$-powerset theories. Some of these examples were derived by previous authors, e.g., S. E. R o d abaugh [16], U. Höhle [13], S. A. Solovyov [18], other examples was presented in $\mathrm{J}$. M o čk o r 12 .

ExAmple 3.1. CSLAT-Powerset theory $\mathcal{P}=(P, \rightarrow, i d, \eta)$ in the category Set, where

(1) $P: \mid$ Set $|\rightarrow| C S L A T \mid$ is defined by $P(X)=\left(2^{X}, \subseteq\right)$,

(2) for each $f: X \rightarrow Y$ in Set, $f_{P}: P(X) \rightarrow P(Y)$ is defined by $f_{P}(S)=f(S)$,

(3) for each $f: X \rightarrow Y$ in Set, $f_{P}^{\leftarrow}: P(Y) \rightarrow P(X)$ is defined by $f_{P}^{\leftarrow}(T)=f^{-1}(T)$

(4) for each $X \in$ Set, $\eta_{X}: X \rightarrow P(X)$ is defined by $\eta_{X}(a)=\{a\}$.

ExAmple 3.2. CSLAT-Powerset theory $\mathbf{Z}=(Z, \rightarrow, i d, \chi)$ in the category Set, where

(1) $Z: \mid$ Set $|\rightarrow| C S L A T \mid$ is defined by $Z(X)=Q^{X}$, where $Q$ is a complete residuated lattice,

(2) for each $f: X \rightarrow Y$ in Set, $f_{Z}: Q^{X} \rightarrow Q^{Y}$ is defined by $f_{Z}(s)(y)=\bigvee_{x \in X, f(x)=y} s(x)$,

(3) for each $f: X \rightarrow Y$ in Set, $f_{Z}^{\leftarrow}: Z(Y) \rightarrow Z(X)$ is defined by $f_{Z}^{\leftarrow}(t)=t \circ f$

(4) for each $X \in$ Set, $\chi_{X}: X \rightarrow Q^{X}, \chi_{X}(\{a\})$ is the characteristic map of a subset $\{a\}$ in a set $X$. 
Example 3.3. CSLAT-Powerset theory $\mathbf{D}=(D, \rightarrow, i d, \rho)$ in the category Set, where

(1) $D: \mid$ Set $|\rightarrow| C S L A T \mid$ is defined by $D(X)=$ the set of all cut systems $\left(C_{\alpha}\right)_{\alpha \in Q}$ in a set $X$, naturally ordered by inclusion,

(2) for each $f: X \rightarrow Y$ in Set, $f_{D}: D(X) \rightarrow D(Y)$

is defined by $f_{D}\left(\left(C_{\alpha}\right)_{\alpha}\right)=\left(\overline{f\left(C_{\alpha}\right)}\right)_{\alpha} \in D(Y)$, where the closure $\left(\overline{S_{\alpha}}\right)_{\alpha}$ in a set $Y$ is defined by $\overline{S_{\alpha}}=\left\{a \in Y: \bigvee_{\beta: a \in C_{\beta}} \beta \geq \alpha\right\}$,

(3) for each $f: X \rightarrow Y$ in Set, $f_{D}^{\leftarrow}: D(Y) \rightarrow D(X)$

is defined by $f_{\mathbf{D}}^{\leftarrow}\left(\left(S_{\alpha}\right)_{\alpha}\right)=\left(f^{-1}\left(S_{\alpha}\right)\right)_{\alpha}$,

(4) for each $X \in$ Set, $\rho_{X}: X \rightarrow D(X)$

is defined by $\rho_{X}(a)=(\{a\})_{\alpha}$.

EXAmple 3.4. CSLAT-Powerset theory $\mathcal{R}=(R, \rightarrow, V, \eta)$ in the category $\operatorname{Set}(Q)$, where

(1) $R:|\operatorname{Set}(Q)| \rightarrow|C S L A T|$ is defined by $R(X, \delta)=P(X)$,

(2) for each morphism $f:(X, \delta) \rightarrow(Y, \gamma)$ in $\operatorname{Set}(Q), f_{R}: R(X, \delta) \rightarrow R(Y, \gamma)$ is defined by $f_{R}(S)=f_{P}(S)$,

(3) for each morphism $f:(X, \delta) \rightarrow(Y, \gamma)$ in $\operatorname{Set}(Q), f_{R}^{\leftarrow}: R(Y, \gamma) \rightarrow R(X, \delta)$ is defined by $f_{R}^{\leftarrow}(T)=f_{P}^{\leftarrow}(T)$,

(4) $V: \operatorname{Set}(Q) \rightarrow$ Set is the forgetfull functor, $V(A, \delta)=A, V(f)=f$,

(5) for each $(X, \delta) \in \operatorname{Set}(Q), \eta_{(X, \delta)}: X \rightarrow R(X)$ is defined by $\eta_{(X, \delta)}(a)=\{a\}$.

ExAmple 3.5. CSLAT-Powerset theory $\mathcal{F}=(F, \rightarrow, V, \widehat{\chi})$ in the category $\operatorname{Set}(Q)$, where

(1) $F:|\operatorname{Set}(Q)| \rightarrow|C S L A T|$ is defined by

$$
F(X, \delta)=\left(H_{\operatorname{Som}(Q)}((X, \delta),(Q, \leftrightarrow)), \leq\right),
$$

(2) for each morphism $f:(X, \delta) \rightarrow(Y, \gamma)$ in $\operatorname{Set}(Q), f_{F}: F(X, \delta) \rightarrow F(Y, \gamma)$ is defined by $f_{F}(s)(y)=\bigvee_{x \in X} s(x) \otimes \gamma(f(x), y)$,

(3) for each morphism $f:(X, \delta) \rightarrow(Y, \gamma)$ in $\operatorname{Set}(Q), f_{F}^{\overleftarrow{F}}: F(Y, \gamma) \rightarrow F(X, \delta)$ is defined by $f_{F}^{\leftarrow}(t)=t \circ f$.

(4) $V: \operatorname{Set}(Q) \rightarrow$ Set is the forgetfull functor,

(5) for each $(X, \delta) \in \operatorname{Set}(Q), \widehat{\chi}_{(X, \delta)}: X \rightarrow F(X, \delta)$

is defined by $\widehat{\chi}_{(X, \delta)}(a)(x)=\delta(a, x)$, for $a, x \in X$.

ExAmple 3.6. $C S L A T$-Powerset theory $\mathcal{C}=(C, \rightarrow, V, \widetilde{\chi})$ in the category $\operatorname{Set}(Q)$, where

(1) $C:|\operatorname{Set}(Q)| \rightarrow|C S L A T|$ is defined by $C(X, \delta)=$ set of all $\mathrm{f}$-cut systems in $(A, \delta)$ in the category $\operatorname{Set}(Q)$, naturally ordered by inclusion, 


\section{CLOSURE THEORIES OF POWERSET THEORIES}

(2) for each morphism $f:(X, \delta) \rightarrow(Y, \gamma)$ in $\operatorname{Set}(Q), f_{C}: C(X, \delta) \rightarrow C(Y, \gamma)$ is defined by

$$
f_{C}\left(\left(E_{\alpha}\right)_{\alpha}\right)=\left(\widetilde{\left.f\left(E_{\alpha}\right)\right)_{\alpha}}, \quad \widetilde{f\left(E_{\alpha}\right)}=\left\{b \in \underset{(y, \beta): y \in f\left(E_{\beta}\right)}{b}, \bigvee_{(b, y) \geq \alpha} \beta \otimes \gamma,\right.\right.
$$

(3) for each morphism $f:(X, \delta) \rightarrow(Y, \gamma)$ in $\operatorname{Set}(Q), f_{C}^{\leftarrow}: C(Y, \gamma) \rightarrow C(X, \delta)$ is defined by $f_{C}^{\leftarrow}\left(\left(E_{\alpha}\right)_{\alpha}\right)=\left(f^{-1}\left(E_{\alpha}\right)\right)_{\alpha}$,

(4) $V: \operatorname{Set}(Q) \rightarrow$ Set is the forgetfull functor,

(5) for each $(X, \delta) \in \operatorname{Set}(Q), \widetilde{\chi}_{(X, \delta)}: X \rightarrow C(X, \delta)$ is defined by $\tilde{\chi}_{(X, \delta)}(a)=(\widetilde{\{a\}})_{\alpha}$, where $\widetilde{\{a\}_{\alpha}}=\{b \in X: \delta(a, b) \geq \alpha\}$.

S. E. Rod a b a u h [16 introduced the notion of a topological theory $\mathbf{T}_{\mathbf{K P}}$ of a powerset theory $\mathbf{P}$ in a ground category $\mathbf{K}$ which extends powerset theory $\mathbf{P}$ in such a way that, roughly speaking, objects of $\mathbf{T}_{\mathbf{K P}}$ are pairs $(A, \tau)$, where $A$ is an object of a powerset theory $\mathbf{P}$ and $\tau$ is a topology $\tau \subseteq P(A)$ such that morphisms $f_{\mathbf{P}}:(P(A), \tau) \rightarrow(P(B), \sigma)$ are continuous. He then proved that topological theory $\mathbf{T}_{\mathbf{K P}}$ of a powerset theory $\mathbf{P}$ in a ground category $\mathbf{K}$ is in fact a topological category. (For a notion of a topological category or topological construct see, e.g., [13.)

In this section we introduce a notion of a closure theory of a powerset theory, which is weaker than the notion of a topological theory. We begin the section with the definition of the closure theory of a powerset theory in the ground category which mostly will be the category Set or the category $\operatorname{Set}(Q)$ of sets with similarity relations. We will use the category $C S L A T$ of complete $\bigvee$-semilatices with complete $\bigvee$-preserving maps as morphisms.

Definition 3.2. Let $\mathbf{K}$ be a ground category and let $\mathbf{T}=(T, \rightarrow, V, \eta)$ be a $C S L A T$-powerset theory in $\mathbf{K}$. Then a closure theory of a powerset theory $\mathbf{T}$ in a ground category $\mathbf{K}$ is a collection $\mathbf{K}[\mathbf{T}]$ of objects and morphisms satisfying the following axioms:

(1) objects in $\mathbf{K}[\mathbf{T}]$ are pairs $(A, c)$, such that

(i) $A$ is an object in $\mathbf{K}$,

(ii) $c$ is a closure operator on an ordered set $T(A) \in C S L A T$,

(2) $f:(A, c) \rightarrow(B, d)$ is a morphism in $\mathbf{K}[\mathbf{T}]$, if

(i) $f: A \rightarrow B$ is a morphism in $\mathbf{K}$,

(ii) $f_{T}:(T(A), c) \rightarrow(T(B), d)$ is continuous in CSLAT, i.e., $f_{T}(c(x)) \leq d\left(f_{T}(x)\right)$.

(3) Composition of morphisms is inherited from $\mathbf{K}$, i.e., if $f:(A, c) \rightarrow(B, d)$, $g:(B, d) \rightarrow(C, e)$, then $g \circ f:(A, c) \rightarrow(C, e)$.

(4) Identities are inherited from $\mathbf{K}$, i.e., for each $(A, c) \in \mathbf{K}[\mathbf{T}], i d_{A}:(A, c) \rightarrow$ $(A, c)$ in $\mathbf{K}[\mathbf{T}]$. 
It is then clear that $T: \mathbf{K} \rightarrow C S L A T$ is a functor such that $T(f)=f_{T}$ and $\mathbf{K}[\mathbf{T}]$ is a category.

Using examples of powerset theories presented above, we can present basic examples of closure theories of powerset theories in the ground category Set or the category $\operatorname{Set}(Q)$.

EXAMPLE 3.7. The following are examples of closure theories of powerset theories in the ground category $\operatorname{Set}$ or $\operatorname{Set}(Q)$.

(1) Closure theory of powerset theory $\operatorname{Set}[\mathcal{P}]$ in the category Set,

(2) Closure theory of powerset theory Set $[\mathbf{Z}]$ in the category Set,

(3) Closure theory of powerset theory Set[D] in the category Set,

(4) Closure theory of powerset theory $\operatorname{Set}(Q)[\mathcal{R}]$ in the category $\operatorname{Set}(Q)$,

(5) Closure theory of powerset theory $\operatorname{Set}(Q)[\mathcal{F}]$ in the category $\operatorname{Set}(Q)$,

(6) Closure theory of powerset theory $\operatorname{Set}(Q)[\mathcal{C}]$ in the category $\operatorname{Set}(Q)$.

The following theorem extends in some way results of S. E. R o d a b a u g h [16] about topological theories of powerset theories.

TheOREM 3.1. Let $\mathbf{K}$ be a topological construct with respect to a forgetfull functor $U: \mathbf{K} \rightarrow$ Set and let $\mathbf{T}=(T, \rightarrow, U, \eta)$ be a CSLAT-powerset theory in a ground category $\mathbf{K}$. Then the closure theory $\mathbf{K}[\mathbf{T}]$ of a powerset theory $\mathbf{T}$ in a ground category $\mathbf{K}$ is also a topological construct.

Proof. Let $V: \mathbf{K}[\mathbf{T}] \rightarrow$ Set be a forgetfull functor such that $V(A, d)=U(A)$, $V(f)=f, U(g)=g$, where $(A, d) \in \mathbf{K}[\mathbf{T}], g$ is a morphism in $\mathbf{K}$ and $f$ is a morphism in $\mathbf{K}[\mathbf{T}]$. Let $X \in \mid$ Set $\mid$ and let $f_{i}: X \rightarrow V\left(A_{i}, d_{i}\right), i \in I$, be a $V$-structured source from $X$. Since $f_{i}, i \in I$, is also a $U$-structured source from $X$, it follows that in a topological construct $\mathbf{K}$, there exists the unique initial lift $g_{i}: Y \rightarrow A_{i}, i \in I$ and a bijection $g: U(Y) \rightarrow X$, such that $U\left(g_{i}\right)=f_{i} \circ g$ for each $i$. For each $i \in I$, the closure $c_{i}$ on $T(Y)$ is defined by

$$
s \in T(Y), \quad c_{i}(s)=g_{i, T}^{\leftarrow}\left(d_{i}\left(g_{i, T}(s)\right)\right)
$$

We have $c_{i}(s)=g_{i, T}^{\overleftarrow{ }}\left(d_{i}\left(f_{i, T}(s)\right)\right) \geq g_{i, T}^{\overleftarrow{ }}\left(g_{i, T}(s)\right) \geq s$. Further, we have

$$
\begin{aligned}
c_{i} c_{i}(s) & =f_{i, T}^{\leftarrow}\left(d_{i}\left(f_{i, T}(c(s))\right)\right) \\
& =f_{i, T}^{\leftarrow}\left(d_{i}\left(f_{i, T} \overrightarrow{(}\left(d_{i}\left(f_{i,} \rightarrow(s)\right)\right)\right)\right) \leq f_{i, T}^{\leftarrow}\left(d_{i} d_{i}\left(f_{i, T}(s)\right)\right)=c(s) .
\end{aligned}
$$

Hence, $c_{i}$ is a closure operator on $T(Y)$. We set $c=\bigwedge_{i \in I} c_{i}$. Clearly, $c(s) \geq s$ and we have $c c(s)=\bigwedge_{i \in I} c_{i}\left(\bigwedge_{j \in I} c_{j}(s)\right) \leq \bigwedge_{i \in I} c_{i} c_{i}(s)=c(s)$. Hence $c$ is a closure 


\section{CLOSURE THEORIES OF POWERSET THEORIES}

operator on $T(Y)$. We show that $g_{i}:(Y, c) \rightarrow\left(A_{i}, d_{i}\right)$ is an initial lift.

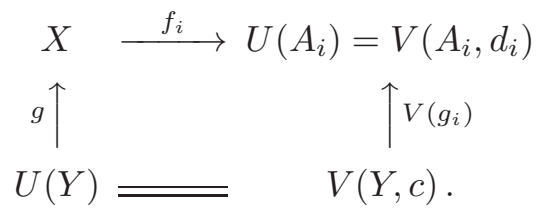

In fact, for $s \in T(Y)$, we have $\overrightarrow{g_{i, T}}(c(s))=\overrightarrow{g_{i, T}}\left(g_{i, T}^{\overleftarrow{ }}\left(d_{i}\left(g_{i, T} \vec{T}(s)\right)\right)\right) \leq d_{i}\left(g_{i, T} \overrightarrow{h_{i}}(s)\right)$, and $g_{i, T}$ is continuous. Now let $(Z, w) \in \mathbf{K}[\mathbf{T}]$ with morphisms $h_{i}:(Z, w) \rightarrow$ $\left(A_{i}, d_{i}\right)$ in $\mathbf{K}[\mathbf{T}]$ and a map $h: V(Z, w) \rightarrow X$ be such that $f_{i} \circ h=V\left(h_{i}\right), i \in I$. Because $Y$ is an initial lift of $f_{i}, i \in I$ in a category $\mathbf{K}$, there exists morphism $n: Z \rightarrow Y$ in $\mathbf{K}$, such that $U(n) \circ g=h, V\left(g_{i}\right) \circ U(n)=V\left(h_{i}\right), i \in I$. We show that in that case, $n_{T}:(T(Z), w) \rightarrow\left(T(Y), c_{i}\right)$ is continuous for every $i \in I$. In fact, since $h_{i}$ is continuous, we have $\overrightarrow{g_{i, T}}\left(n_{T} \vec{T}(w(s))\right)=h_{i, T}(w(s)) \leq d_{i}\left(\overrightarrow{h_{i, T}}(s)\right)$. Then we obtain

$$
\begin{aligned}
& n_{T}(w(s)) \leq g_{i, T}^{\leftarrow}\left(g_{i, T}\left(n_{T}(w(s))\right)\right) \\
& \leq g_{i, T}^{\overleftrightarrow{\leftrightarrow}}\left(d_{i}\left(h_{i, T}(s)\right)\right)=g_{i, T}^{\overleftarrow{ }}\left(d_{i}\left(g_{i} \circ n\right)_{i, T}(s)\right)=c_{i}\left(n_{T}(s)\right)
\end{aligned}
$$

It is then clear that $n_{T}:(T(Z), w) \rightarrow(T(Y), c)$ is also continuous and that $(Y, c)$ is the unique lift. Hence, $\mathbf{K}[\mathbf{T}]$ is a topological construct.

COROLlaRY 3.1. Each closure theory of a powerset theory from Example 3.7 is a topological construct.

P r o of. We need to prove only that $\operatorname{Set}(Q)$ is a topological construct. Let $U: \operatorname{Set}(Q) \rightarrow$ Set be the forgetfull functor such that $U(A, \delta)=A, U(f)=f$ and let $f_{i}: X \rightarrow U\left(A, \delta_{i}\right), i \in I$ be a $U$-structured source from $X$. Then $(X, \delta)$ with $\delta(x, y)=\bigwedge_{i \in I} \delta_{i}(x, y)$, is an initial lift and $\operatorname{Set}(Q)$ is a topological construct.

\section{Closure theories of powerset theories in the category Set}

In this section we deal with properties of objects of closure theories of powerset theories in the category Set. An important notion in the section will be the notion of a closure operator which preserves a canonical form of fuzzy objects. Using that notion we will characterize some extensions of closure operators defined on different powerset objects in the category Set. Moreover, using that notion we show that a closure theory of a classical powerset theory in the ground category Set is a coreflective subcategory in the subcategory of closure theory 
of (Zadeh's) powerset theory, which is based on closure operators which preserves a canonical forms of (Zadeh's) fuzzy sets.

In the paper [14] it was proved that any fuzzy set $s \in Z(A)$ can be expressed in the following canonical form, where $\underline{\alpha}$ is a constant fuzzy set with the constant value $\alpha$ :

$$
s=\bigvee_{\alpha \in Q} \underline{\alpha} \otimes \chi\left(s_{\alpha}\right),
$$

where $\chi=\chi_{A}: P(A) \rightarrow Z(A)$ is the characteristic map, i.e., $\chi_{A}(X)(a)=1_{Q}$ if and only if $a \in X$, and $0_{Q}$, otherwise. Sometimes instead of $\chi_{A}(X)$ we write $\chi(X)$ only.

Let us consider the full subcategory $\operatorname{Set}[Z]_{0}$ of the category $\operatorname{Set}[Z]$ with objects $(A, d)$, such that a closure operator $d$ preserves canonical form, i.e., for any fuzzy set $s \in Z(A)$, the following condition holds:

$$
d(s)=\bigvee_{\alpha \in Q} \underline{\alpha} \otimes d\left(\chi\left(s_{\alpha}\right)\right) .
$$

Proposition 4.1. Let $(A, c) \in \operatorname{Set}[P]$. Then $\left(A, \psi_{A, Z(A)}(c)\right) \in \operatorname{Set}[Z]_{0}$.

Proof. For simplicity we set $d=\psi_{A, Z(A)}(c)$. Let $s \in Z(A)$. It is clear that

$$
d(s)=d\left(\bigvee_{\alpha \in Q} \underline{\alpha} \otimes \chi\left(s_{\alpha}\right)\right) \geq \bigvee_{\alpha \in Q} d\left(\underline{\alpha} \otimes \chi\left(s_{\alpha}\right)\right) .
$$

Now, for any $\beta \in Q, \beta>0$, we have

$$
\left(\underline{\alpha} \otimes \chi\left(s_{\alpha}\right)\right)_{\beta}=\left\{x \in A: \alpha \otimes \chi\left(s_{\alpha}\right)(x) \geq \beta\right\}=s_{\alpha},
$$

if $\beta \leq \alpha$, or $\emptyset$, if $\beta \not \alpha \alpha$. It follows that

$$
d\left(\underline{\alpha} \otimes \chi\left(s_{\alpha}\right)\right)(a)=\bigvee_{\beta, a \in c\left(\left(\underline{\alpha} \otimes \chi\left(s_{\alpha}\right)\right)_{\beta}\right)}=\bigvee_{\beta, \beta \leq \alpha, a \in c\left(s_{\alpha}\right)} \beta=\alpha \otimes \chi\left(c\left(s_{\alpha}\right)\right)(a)
$$

On the other hand, we have $\underline{\alpha}_{\beta}=\{x \in A: \alpha \geq \beta\}=A$, if $\beta \leq \alpha$, or $\emptyset$, otherwise. It follows that

$$
d(\underline{\alpha})(a)=\bigvee_{\beta, a \in c\left(\underline{\alpha}_{\beta}\right)} \beta=\bigvee_{\beta, \beta \leq \alpha, a \in A} \beta=\alpha .
$$

Further, we have $\chi\left(s_{\alpha}\right)_{\beta}=\left\{x \in A: \chi\left(s_{\alpha}\right)(x) \geq \beta\right\}=s_{\alpha}$, and it follows that

$$
d\left(\chi\left(s_{\alpha}\right)\right)(a)=\bigvee_{\beta, a \in c\left(\chi\left(s_{\alpha}\right)_{\beta}\right)}=\bigvee_{\beta, a \in c\left(s_{\alpha}\right)} \beta=\chi\left(c\left(s_{\alpha}\right)\right)(a) .
$$

Hence, we obtain

$$
d(\underline{\alpha})(a) \otimes d\left(\chi\left(s_{\alpha}\right)\right)(a)=\alpha \otimes \chi\left(c\left(s_{\alpha}\right)\right)(a)=d\left(\underline{\alpha} \otimes \chi\left(s_{\alpha}\right)\right)(a),
$$


and it follows that

$$
\begin{aligned}
d(s)=d\left(\bigvee_{\alpha \in Q} \underline{\alpha} \otimes \chi\left(s_{\alpha}\right)\right) & \\
& \geq \bigvee_{\alpha \in Q} d\left(\underline{\alpha} \otimes \chi\left(s_{\alpha}\right)\right)=\bigvee_{\alpha \in Q} \underline{\alpha} \otimes \chi\left(c\left(s_{\alpha}\right)\right)=\bigvee_{\alpha \in Q} \underline{\alpha} \otimes d\left(\chi\left(s_{\alpha}\right)\right) .
\end{aligned}
$$

Now we show that $d(s) \leq \bigvee_{\alpha \in Q} \underline{\alpha} \otimes d\left(\chi\left(s_{\alpha}\right)\right)$. In fact, for any $a \in A$, we have

$$
\begin{gathered}
d(s)(a)=\bigvee_{\beta, a \in c\left(s_{\beta}\right)} \beta \\
\bigvee_{\alpha \in Q} \underline{\alpha}(a) \otimes d\left(\chi\left(s_{\alpha}\right)\right)(a)=\bigvee_{\alpha \in Q} \alpha \otimes\left(\begin{array}{c}
\bigvee_{\gamma, a \in c\left(\chi\left(s_{\alpha}\right)_{\gamma}\right)} \\
\gamma
\end{array}\right)=\bigvee_{\alpha \in Q} \alpha \otimes\left(\bigvee_{\gamma, a \in c\left(s_{\alpha}\right)} \gamma\right)=(*) .
\end{gathered}
$$

If $\beta \in Q$ is such that $a \in c\left(s_{\beta}\right)$, then we have

$$
(*) \geq \beta \otimes\left(\bigvee_{\gamma, a \in c\left(s_{\beta}\right)} \gamma\right)=\beta \otimes 1_{Q}=\beta,
$$

and it follows that $(*) \geq \bigvee_{\beta, a \in c\left(s_{\beta}\right)} \beta=d(s)(a)$.

TheOREM 4.1. There exist adjoint functors

$$
\operatorname{Set}[P] \underset{H}{\stackrel{G}{\rightleftarrows}} \operatorname{Set}[Z]_{0} .
$$

P r o of. Let $f:(A, c) \rightarrow(B, d)$ be a morphism in $\operatorname{Set}[P]$. The object function of $G$ is defined by $G(A, c)=\left(A, \psi_{A, Z(A)}(c)\right)$ and $G(f)=f$. According to Property 4.1, $G(A, c) \in \operatorname{Set}[Z]_{0}$ and according to Property $2.1, f_{Z}=Z(f)$ : $\left(Z(A), \psi_{A, Z(A)}(c)\right) \rightarrow\left(Z(B), \psi_{B, Z(B)}(d)\right)$ is continuous and $G$ is a functor. The functor $H$ is defined symmetrically, i.e., for any continuous morphism $g:(A, c) \rightarrow(B, d)$ in $\operatorname{Set}[Z]_{0}$, the object function is defined by $H(A, c)=$ $\left(A, \psi_{Z(A), A}(c)\right)$ and $H(g)=g$. We show that $g_{P}=P(g):\left(P(A), \psi_{Z(A), A}(c)\right) \rightarrow$ $\left(P(B), \psi_{Z(B), B}(d)\right)$ is continuous.

In fact, we need to prove that if $g$ is continuous, i.e., $g_{Z}(c(s)) \leq d\left(g_{\vec{Z}}(s)\right)$, for any $s \in Z(A)$, for any $X \subseteq A$,

$$
g\left(\operatorname{core} c\left(\chi_{X}\right)\right)=g_{P}\left(\psi_{Z(A), A}(c)(X)\right) \subseteq \psi_{Z(B), B}(d)\left(g_{P}(X)\right)=\text { core } d\left(\chi_{g(X)}\right)
$$

holds. Let $a \in$ core $c\left(\chi_{X}\right)$, i.e., $c\left(\chi_{X}\right)(a)=1_{Q}$. From the continuity of $g_{Z}$ it follows, that

$$
1_{Q}=c\left(\chi_{X}\right)(a)=\bigvee_{x, g(x)=g(a)} c\left(\chi_{X}\right)(x)=g_{Z}\left(c\left(\chi_{X}\right)(g(a))\right) \leq d\left(g_{Z}\left(\chi_{X}\right)\right)(g(a)) .
$$


Since $g_{Z}\left(\chi_{X}\right)=\chi_{g(X)}$, we obtain that $d\left(\chi_{g(X)}\right)(g(a))=d\left(g_{Z}\left(\chi_{X}\right)\right)(g(a))=1_{Q}$ and it follows that $g(a) \in$ core $d\left(\chi_{g(X)}\right)$. Hence, $g_{\mathbf{P}}$ is continuous and $H$ is a functor.

We prove that there exist natural transformations

$$
\begin{aligned}
\varepsilon: G \circ H & \rightarrow 1_{\operatorname{Set}[Z]_{0}}, \\
\tau: 1_{\operatorname{Set}[P]} & \rightarrow H \circ G .
\end{aligned}
$$

According to Theorem 2.1, for any object $(A, c) \in \operatorname{Set}[P], H \circ G(A, c)=(A, c)$ and we can set $\tau_{(A, c)}=1_{A}$ in the category $\operatorname{Set}[P]$ and $\tau$ is a natural transformation. Now, for $(A, c) \in \operatorname{Set}[Z]_{0}$, we set $\varepsilon_{(A, c)}=1_{A}$ in the category $\operatorname{Set}[Z]_{0}$, i.e.,

$$
\varepsilon_{(A, c)}=1_{A}: G \circ H(A, c)=\left(A, \psi_{A, Z(A)} \circ \psi_{Z(A), A}(c)\right) \rightarrow(A, c) .
$$

We need only to prove that $Z\left(1_{A}\right)$ is continuous, i.e., for any $s \in Z(A), \psi_{A, Z(A)} \circ$ $\psi_{Z(Z), A}(c)(s) \leq c(s)$. For every $a \in A$, we have

$$
\psi_{A, Z(A)} \circ \psi_{Z(A), A}(c)(s)(a)=\bigvee_{\left\{\beta: a \in \psi_{Z(A), A}(c)\left(s_{\beta}\right)\right\}}=\bigvee_{\left\{\beta: c\left(\chi\left(s_{\beta}\right)\right)(a)=1\right\}} \beta .
$$

According to [14, $s$ can be expressed by $s=\bigvee_{\alpha \in Q} \underline{\alpha} \otimes \chi\left(s_{\alpha}\right)$. Let $\beta \in Q$ be such that $c\left(\chi\left(s_{\beta}\right)\right)(a)=1_{Q}$. Since the closure operator $c$ preserves the canonical form, we have

$$
\begin{aligned}
c(s)(a)=c\left(\bigvee_{\alpha \in Q} \underline{\alpha} \otimes \chi\left(s_{\alpha}\right)\right)(a) & \geq \bigvee_{\alpha \in Q} \underline{\alpha}(a) \otimes c\left(\chi\left(s_{\alpha}\right)\right)(a) \\
& \geq \underline{\beta}(a) \otimes c\left(\chi\left(s_{\beta}\right)\right)(a)=\beta .
\end{aligned}
$$

It follows that $c(s)(a) \geq \bigvee_{\left\{\beta: c\left(\chi\left(s_{\beta}\right)\right)(a)=1_{Q}\right\}} \beta=\psi_{A, Z(A)} \circ \psi_{Z(Z), A}(c)(s)(a)$, and $\varepsilon_{(A, c)}$ is continuous. Moreover, it is clear that the following compositions are identities:

$$
\begin{aligned}
& H \stackrel{\tau H}{\longrightarrow} H \circ G \circ H \stackrel{G \varepsilon}{\longrightarrow} H, \\
& G \stackrel{G \tau}{\longrightarrow} G \circ H \circ G \stackrel{\varepsilon G}{\longrightarrow} G .
\end{aligned}
$$

Hence, $H$ and $G$ are adjoint functors.

TheOrem 4.2. Set $[P]$ is a coreflective subcategory in the category $S e t[Z]_{0}$.

For the proof of the theorem we need the following proposition.

Proposition 4.2. Let $A, B$ be sets and let $c$ (d, respectively) be a closure operator on $Z(A)(Z(B)$, respectively). Let $f: A \rightarrow B$ be a map such that $Z(f):(Z(A), c) \rightarrow(Z(B), d)$ be continuous, Then

$$
P(f):\left(P(A), \psi_{Z(A), A}(c)\right) \rightarrow\left(P(B), \psi_{Z(B), B}(d)\right)
$$

is continuous. 


\section{CLOSURE THEORIES OF POWERSET THEORIES}

Pr o of. We need to prove that

$$
f\left(\operatorname{core} c\left(\chi_{X}\right)\right)=f\left(\psi_{Z(A), A}(c)(X)\right) \subseteq \psi_{Z(B), B}(d)(f(X))=\text { core } d\left(\chi_{f(X)}\right),
$$

for any $X \subseteq A$. Suppose that it is not true. Then there exists $a \in \operatorname{core} c\left(\chi_{X}\right)$ such that $f(a) \notin$ core $d\left(\chi_{f(X)}\right)$. Hence, $c\left(\chi_{X}\right)(a)=1_{Q}, d\left(\chi_{f(X)}\right)(f(a))<1_{Q}$. Since $Z(f)$ is continuous, for $\chi_{X} \in Z(A)$ we have $Z(f)\left(c\left(\chi_{X}\right)\right) \leq d\left(Z(f)\left(\chi_{X}\right)\right)$. Then for $f(a)$ we obtain

$$
Z(f)\left(c\left(\chi_{X}\right)\right)(f(a))=\bigvee_{f(x)=f(a)} c\left(\chi_{X}\right)(x) \geq c\left(\chi_{X}\right)(a)=1_{Q},
$$

and it follows that $d\left(Z(f)\left(\chi_{X}\right)\right)(f(a))=1_{Q}$. On the other hand, since

$$
Z(f)\left(\chi_{X}\right)(f(a))=\bigvee_{f(x)=f(a)} \chi_{X}(x)=\chi_{f(X)}(f(a)),
$$

we obtain $1_{Q}=d\left(Z(f)\left(\chi_{X}\right)\right)(f(a))=d\left(\chi_{f(X)}\right)(f(a))<1_{Q}$, a contradiction.

Proof of Theorem. Since $G$ is a left adjoint of the functor $H$, to prove that $\operatorname{Set}[P]$ is a coreflective subcategory in $\operatorname{Set}[Z]_{0}$ we need to prove only that the functor $G$ is full and faithfull. Hence, we need to show that for arbitrary objects $(A, c),(B, d)$ from the category $\operatorname{Set}[P]$,

$$
G: \operatorname{Hom}_{\operatorname{Set}[P]}((A, c),(B, d)) \rightarrow \operatorname{Hom}_{\text {Set }[Z]_{0}}(G(A, c), G(B, d))
$$

is a bijection. Let $f: G(A, c) \rightarrow G(B, d)$ be a morphism in $\operatorname{Set}[Z]_{0}$, i.e., $f: A \rightarrow B$ is a map such that $Z(f):\left(Z(A), \psi_{A, Z(A)}(c)\right) \rightarrow\left(Z(B), \psi_{A, Z(A)}(d)\right)$ is continuous. Then according to Proposition 2.2 and Theorem 2.1,

$$
P(f):\left(P(A), c=\psi_{Z(A), A} \psi_{A, Z(A)}(c)\right) \rightarrow\left(P(B), d=\psi_{Z(B), B} \psi_{B, Z(B)}(d)\right)
$$

is continuous and it follows that $f:(A, c) \rightarrow(B, d)$ is a morphism in the category $\operatorname{Set}[P], G(f)=f$. Hence, $G$ is full and it is clear that $G$ is also faithfull.

In the following two propositions we internally characterize closure operators $\psi_{Z(A), A}(c)$ and $\psi_{A, Z(A)}(d)$.

Proposition 4.3. Let $(A, c) \in \operatorname{Set}[Z]$. Then $\psi_{Z(A), A}(c)$ is the largest closure operatord on $(P(A), \subseteq)$, such that

$$
\chi:(P(A), d) \rightarrow(Z(A), c)
$$

is continuous, where $\chi$ is the characteristic map.

Proof. Let $X \in P(A)$. We show that

$$
\chi:\left(P(A), \psi_{Z(A), A}(c)\right) \rightarrow(Z(A), c)
$$

is continuous. Since

$$
\chi\left(\psi_{Z(A), A}(c)(X)\right)(a)=1_{Q} \quad \text { if and only if } \quad c(\chi(X))(a)=1_{Q},
$$


otherwise $\chi\left(\psi_{Z(A), A}(c)(X)\right)(a)=0_{Q}$, then $\chi\left(\psi_{Z(A), A}(c)(X)\right) \leq c(\chi(X))$ and $\chi$ is continuous. Now, let $d$ be another closure operator on $P(A)$, such that $\chi:(P(A), d) \rightarrow(Z(A), c)$ is continuous. Then for any $X \subseteq A, a \in A, \chi(d(X))(a) \leq$ $c(\chi(X))(a)$. If $a \in d(X)$, then $c(\chi(X))(a)=1_{Q}$ and we have $a \in \psi_{Z(A), A}(c)(X)$. Hence, $d \leq \psi_{Z(A), A}$.

Proposition 4.4. Let $(A, c) \in \operatorname{Set}[P]$. Then $\psi_{A, Z(A)}(c)$ is the smallest closure operator d on $(Z(A), \leq)$ which preserves canonical form and such that

$$
\chi:(P(A), c) \rightarrow(Z(A), d)
$$

is continuous, where $\chi$ is a characteristic map.

P r o o f. According to Proposition 4.1, $d:=\psi_{A, Z(A)}(c)$ preserves canonical form. We show that $\chi:(P(A), c) \rightarrow(Z(A), d)$ is continuous. It follows directly from $d(c)(\chi(X))(a)=\bigvee_{\beta, a \in c\left(\chi(X)_{\beta}\right)} \beta=\bigvee_{\beta, a \in c(X)} \beta=\chi(c(X))(a)$. Now, let $r$ be a closure operator on $Z(A)$ such that $\chi$ is continuous and $r$ preserves canonical form, i.e., for any $s \in Z(A)$, we have $r(s) \geq \bigvee_{\alpha \in Q} \underline{\alpha} \otimes r\left(\chi\left(s_{\alpha}\right)\right)$ and $\chi(c(X)) \leq r(\chi(X))$. Then for any $s \in Z(A)$, we have $\chi\left(c\left(s_{\alpha}\right)\right) \leq r\left(\chi\left(s_{\alpha}\right)\right)$. Then we obtain

$$
\begin{aligned}
& d\left(\chi\left(s_{\alpha}\right)\right)(a)= \psi_{A, Z(A)}(c)\left(\chi\left(S_{\alpha}\right)\right)(a)= \\
& \bigvee_{\beta, a \in c\left(\chi\left(s_{\alpha}\right)_{\beta}\right)} \beta=\bigvee_{\beta, a \in c\left(s_{\alpha}\right)} \beta=\chi\left(c\left(s_{\alpha}\right)\right)(a) \leq r\left(\chi\left(s_{\alpha}\right)\right) .
\end{aligned}
$$

Hence, we obtain

$$
r(s) \geq \bigvee_{\alpha \in Q} \underline{\alpha} \otimes r\left(\chi\left(s_{\alpha}\right)\right) \geq \bigvee_{\alpha \in Q} \underline{\alpha} \otimes d\left(\chi\left(s_{\alpha}\right)\right)=d(s) .
$$

It is clear that the category Set can be considered to be a subcategory of $\operatorname{Set}[P]$ and also Set $[Z]$. In fact, we can introduce embedding functors

$$
1_{P}: \operatorname{Set} \rightarrow \operatorname{Set}[P], \quad 1_{Z}: \operatorname{Set} \rightarrow \operatorname{Set}[Z],
$$

such that for any $A \in$ Set and any map $f: A \rightarrow B$ in Set, $1_{P}(A)=\left(A, 1_{P(A)}\right)$, $1_{P}(f)=f$, and, analogously, $1_{Z}(A)=\left(A, 1_{Z(A)}\right), 1_{Z}(f)=f$, where $1_{P(A)}$ and $1_{Z(A)}$ are considered to be (trivial) closure operators. Then the power objects functors $P$ and $Z$ which are defined as $P, Z$ : Set $\rightarrow C S L A T$ can be extended to the power objects functors defined over categories $\operatorname{Set}[P]$ and $\operatorname{Set}[Z]$ as follows.

Let $C S L A T_{c l}$ be the category with objects $(V, \leq, c)$, where $(V, \leq)$ is a complete $\wedge$-semilattice and let $c$ be a closure operator on $(V, \leq)$. Morphisms in $C S L A T_{c l}$ are continuous, $\wedge$-preserving maps $f:(V, \leq, c) \rightarrow(W, \leq, d)$. Then the functors

$$
P_{c l}: \operatorname{Set}[P] \rightarrow C S L A T_{c l}, \quad Z_{c l}: \operatorname{Set}[Z] \rightarrow C S L A T_{c l}
$$




\section{CLOSURE THEORIES OF POWERSET THEORIES}

are defined as follows.

$$
\begin{aligned}
& (A, c) \in \operatorname{Set}[P], \quad P_{c l}(A, c)=(P(A), \subseteq, c), \quad P_{c l}(f)=f_{P}, \\
& (A, d) \in \operatorname{Set}[Z], \quad Z_{c l}(A, d)=(Z(A), \leq, d), \quad Z_{c l}(f)=f_{Z} .
\end{aligned}
$$

All these functors can be represented by the following commutative diagram.

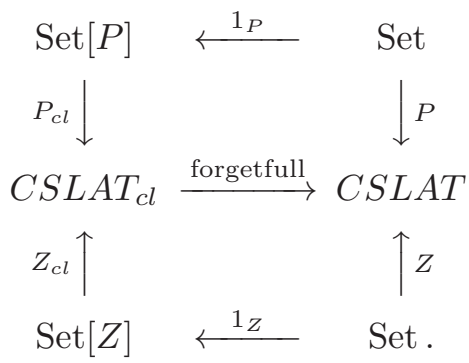

In many papers a relationships between powerset functor $P$ and fuzzy objects functor $Z$ were investigated, see, e.g., [14, 19, 24, 26, 29] for some examples. The basic relationship between functors $P, Z$ can be represented by the fact, that the characteristic maps $\chi_{A}: P(A) \rightarrow Z(A)$ represent natural transformation between these functors, i.e.,

$$
\chi: P \rightarrow Z \text {. }
$$

In the following proposition we extend that relationship to the case of powerset functors with closure operators, i.e., instead of functors $P, Z$ we consider the functors $P_{c l}, Z_{c l}$.

Proposition 4.5. There exists a natural transformation

$$
\chi: P_{c l} \rightarrow Z_{c l} \circ G .
$$

Pr o of. Let $(A, c) \in \operatorname{Set}[P]$. We define

$$
\chi_{(A, c)}:(P(A), \subseteq, c) \rightarrow\left(Z(A), \leq, \psi_{A, Z(A)}(c)\right)
$$

by $\chi_{(A, c)}(X)=\chi(X): A \rightarrow Q$. According to Prop. 4.4, $\chi_{(A, c)}$ is continuous. It is then easy to verify that for any morphism $f:(A, c) \rightarrow(B, d)$ in $\operatorname{Set}[P]$, the following diagram commutes:

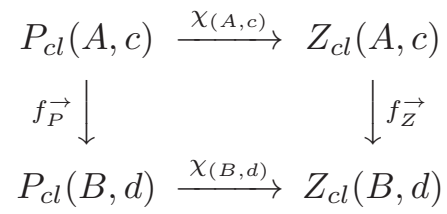

and $\chi$ is a natural transformation.

The functor $D:$ Set $\rightarrow$ Set is a special case of the functor $C: \operatorname{Set}(Q) \rightarrow$ Set (for similarity relations equal to classical identity relation) and from [9], [10], 
it follows that there is a natural equivalence between functors $D$ and $Z, \sigma: D \rightarrow Z$, such that for any set $A, \mathbf{E}=\left(E_{\alpha}\right)_{\alpha} \in D(A)$ and any $s \in Z(A)$, we have

$$
a \in A, \quad \sigma_{A}(\mathbf{E})(a)=\bigvee_{\beta, a \in E_{\beta}} \beta, \quad \sigma_{A}^{-1}(s)=\left(s_{\alpha}\right)_{\alpha} .
$$

Using that result and results from Theorem 2.1, we can prove the following theorem.

Proposition 4.6. The categories Set $[Z]$ and $S e t[D]$ are isomorphic.

Proof. We define a functor $J: \operatorname{Set}[Z] \rightarrow \operatorname{Set}[D]$, such that for any morphism $f:(A, c) \rightarrow(B, d)$ in the category $\operatorname{Set}[Z]$, we put

$$
J(A, c)=\left(A, \psi_{Z(A), D(A)}(c)\right), \quad J(f)=f .
$$

Then according to [1]; Theorem 3.1 and Proposition 4.2,

$$
f:\left(A, \psi_{Z(A), D(A)}(c)\right) \rightarrow\left(B, \psi_{Z(B), D(B)}(d)\right)
$$

is a morphism in Set $[D]$ and the definition of $J$ is correct and, analogously, it can be showed that there exists the inverse functor $I$ such that for any morphism $g:(X, u) \rightarrow(Y, v)$ from the category $\operatorname{Set}[D]$,

$$
I(X, u)=\left(X, \psi_{D(A), Z(A)}(u)\right), \quad I(g)=g .
$$

\section{Closure theories of powerset theories in the category $\operatorname{Set}(Q)$}

In this section we deal with properties of objects of closure theories of powerset theories in the category $\operatorname{Set}(Q)$. An important notion in the section will be again the notion of a closure operator which preserves a canonical form of fuzzy objects. Using that notion we will characterize some extensions of closure operators defined on different powerset objects in the category Set.

In the paper [12, Lemma 2], we proved that any fuzzy object $s \in F(A, \delta)$ can be expressed by the following canonical form, in which we use the closure operator ${ }^{\text {: }}$ :

$$
s=\bigvee_{\alpha \in Q} \underline{\alpha} \otimes \widehat{\chi\left(s_{\alpha}\right)} .
$$

Then we say that a closure operator $d$ defined on $F(A, \delta)$ preserves the canonical form, if for any $s \in F(A, \delta)$, we have

$$
d(s)=\bigvee_{\alpha \in Q} \underline{\alpha} \otimes d\left(\widehat{\chi\left(s_{\alpha}\right)}\right) .
$$




\section{CLOSURE THEORIES OF POWERSET THEORIES}

Let $\operatorname{Set}(Q)[P]_{1}$ be the full subcategory of the category $\operatorname{Set}(Q)[P]$ with objects $((A, \delta), c)$, such that $c$ is continuous with respect to the extension map $\sim$ : $D(A) \rightarrow C(A, \delta)$ (see [11, Def. 3.1]). If we set $w=\psi_{A, Z(A)}(c)$, then $c$ satisfies that property if and only if $w(\widehat{s}) \leq \widehat{w(s)}$, for any $s \in Z(A)$ (see [11, Lemma 3.7]).

Let

$$
\eta_{(A, \delta)}: P(A, \delta) \rightarrow F(A, \delta)
$$

be an extension of the classical characteristic map, i.e., $\eta_{(A, \delta)}(X)=\widehat{\chi(X)}$, for any $X \in P(A, \delta)$. Then the following analogy of Proposition 4.4, holds.

Proposition 5.1. Let $((A, \delta), c) \in \operatorname{Set}(Q)[P]_{1}$. Then $\varphi_{A, F(A, \delta)}(c)$ is the smallest closure operator $d$ on $F(A, \delta)$, which preserves canonical form and such that

$$
\eta_{(A, \delta)}:(P(A, \delta), c) \rightarrow(F(A, \delta), d)
$$

is continuous.

P r o of. We show firstly that $d:=\varphi_{A, F(A, \delta)}(c)$ preserves canonical form. According to [11]; Theorem 3.2, $((A, \delta), d) \in \operatorname{Set}(Q)[F]$ and $d(s)=\psi_{A, Z(A)}(c)(s)$. For simplicity, we put $w=\psi_{A, Z(A)}(c)$. Let $s \in F(A, \delta)$. Then we have

$$
s=\bigvee_{\alpha \in Q} \underline{\alpha} \otimes \widehat{\chi\left(s_{\alpha}\right)} .
$$

According to Proposition 4.1, $w$ preserves canonical form and for any $a \in A$, we obtain

$$
\begin{aligned}
& d(s)(a)=\widehat{w(s)}(a)=w\left(\widehat{\bigvee_{\alpha \in Q} \underline{\alpha} \otimes \widehat{\chi\left(s_{\alpha}\right)}}\right)(a)=\bigvee_{\alpha \in Q} \underline{\underline{\alpha} \otimes w\left(\overline{\chi\left(s_{\alpha}\right)}\right)}(a)= \\
& \bigvee_{x \in A}\left(\bigvee_{\alpha \in Q} \alpha \otimes w\left(\widehat{\chi\left(s_{\alpha}\right)}\right)(x)\right) \otimes \delta(a, x)=\bigvee_{\alpha \in Q}\left(\bigvee_{x \in A} \alpha \otimes w\left(\widehat{\chi\left(s_{\alpha}\right)}\right)(x)\right) \otimes \delta(a, x)= \\
& \bigvee_{\alpha}\left(\underline{\alpha} \otimes w \widehat{\left(\widehat{\chi\left(s_{\alpha}\right)}\right)}\right)(a)=\bigvee_{\alpha \in Q} \underline{\alpha}(a) \otimes d\left(\widehat{\chi\left(s_{\alpha}\right)}\right)(a) .
\end{aligned}
$$

Now we show that $\eta_{(A, \delta)}:(P(A, \delta), c) \rightarrow(F(A, \delta), d)$ is continuous. In fact, for any $X \in P(A, \delta)$ and $a \in A$, we need to prove

$$
\eta_{(A, \delta)}(c(X))(a) \leq d\left(\eta_{(A, \delta)}(X)\right)(a) .
$$


For any $s \in F(A, \delta)$, we have

$$
\begin{gathered}
\left.d\left(\eta_{(A, \delta)}\right)(a)=d(\widehat{\chi(X)})(a)=w \widehat{(\widehat{\chi(X)})}\right)(a)= \\
\bigvee_{x \in A} w(\widehat{\chi(X)})(x) \otimes \delta(a, x)=\bigvee_{x \in A} \bigvee_{\beta, x \in c(\widehat{\chi(X)})} \beta \otimes \delta(a, x) \geq \\
\bigvee_{x \in A} \bigvee_{\beta, x \in c(X)} \beta \otimes \delta(a, x) \geq \bigvee_{x \in c(X)} \delta(a, x)= \\
\bigvee_{x \in A} \chi(c(X)) \otimes \delta(a, x)=\chi \widehat{(c(X))}(a)=\eta_{(A, \delta)}(c(X))(a),
\end{gathered}
$$

since

$$
\widehat{\chi(X)}_{\beta}=\left\{y \in A: \bigvee_{z \in X} \delta(y, z) \geq \beta\right\} \supseteq X,
$$

and, hence, $c(X) \subseteq c\left(\widehat{\chi(X)}_{\beta}\right)$.

Now, let $r$ be another closure operator on $F(A, \delta)$, such that it preserves canonical form and $\eta_{(A, \delta)}:(P(A, \delta), c) \rightarrow(F(A, \delta), r)$ is continuous. Then for any $s \in F(A, \delta)$ and any $\alpha \in Q$, we have $\eta_{(A, \delta)}\left(c\left(s_{\alpha}\right)\right) \leq r\left(\eta_{(A, \delta)}\left(s_{\alpha}\right)\right)$, and we receive

$$
\begin{aligned}
& d\left(\widehat{\chi\left(s_{\alpha}\right)}\right)(a)=d\left(\eta_{(A, \delta)}\left(s_{\alpha}\right)\right)(a)=w\left(\widehat{\left(\eta_{(A, \delta)}\right.}\left(s_{\alpha}\right)\right)(a)= \\
&\left.\left.w \widehat{\left(\widehat{\chi\left(s_{\alpha}\right)}\right)}(a) \leq w \widehat{\left(\chi\left(s_{\alpha}\right)\right.}\right)(a)=w \widehat{\left(\chi\left(s_{\alpha}\right)\right.}\right)(a) .
\end{aligned}
$$

Since

$$
w\left(\chi\left(s_{\alpha}\right)\right)(a)=\bigvee_{\beta, a \in c\left(\chi\left(s_{\alpha}\right)_{\beta}\right)} \beta=\bigvee_{\beta, a \in c\left(s_{\alpha}\right)} \beta=\chi\left(c\left(s_{\alpha}\right)\right),
$$

we obtain

$$
\left.\left.d\left(\widehat{\chi\left(s_{\alpha}\right)}\right)(a) \leq w \widehat{\left(\chi\left(s_{\alpha}\right)\right.}\right)(a)=\chi \widehat{\left(c\left(s_{\alpha}\right)\right.}\right)(a)=\eta_{(A, \delta)}\left(c\left(s_{\alpha}\right)\right) \leq r\left(\widehat{\chi\left(s_{\alpha}\right)}\right)(a) .
$$

It follows that

$$
d(s)=\bigvee_{\alpha \in Q} \underline{\alpha} \otimes d\left(\widehat{\chi\left(s_{\alpha}\right)}\right) \leq \bigvee_{\alpha \in Q} \underline{\alpha} \otimes r\left(\widehat{\chi\left(s_{\alpha}\right)}\right)=r(s) .
$$

Proposition 5.2. Let $((A, \delta), c) \in \operatorname{Set}(Q)[F]$. Then $\varphi_{F(A, \delta), A}(c)$ is the largest closure operator d on $P(A, \delta)$ such that

$$
\eta_{(A, \delta)}:(P(A, \delta), d) \rightarrow(F(A, \delta), c)
$$

is continuous. 


\section{CLOSURE THEORIES OF POWERSET THEORIES}

P r o of. To be $\eta_{(A, \delta)}$ continuous, we need to prove

$$
\eta_{(A, \delta)}\left(\varphi_{F(A, \delta), A}(c)(X)\right)(a) \leq c\left(\eta_{(A, \delta)}(X)\right)(a), \quad \text { for any } X \subseteq A, a \in A .
$$

We have

$$
\begin{aligned}
\eta_{(A, \delta)}\left(\varphi_{F(A, \delta), A}(c)\right)(a) & =\chi\left(\widehat{\varphi_{F(A, \delta), A}}(c)(X)\right)(a)= \\
\bigvee_{x \in A} \chi\left(\varphi_{F(A, \delta), A}(c)(X)\right)(x) \otimes \delta(a, x) & =\bigvee_{x \in A} \chi(\operatorname{core} c(\widehat{\chi(X)}))(x) \otimes \delta(a, x)= \\
\bigvee \delta(a, x) & =\bigvee c(\widehat{\chi(X)})(x) \otimes \delta(a, x) \leq \\
x, c(\widehat{\chi(X)})(x)=1 \quad x, c(\widehat{\chi(X)})(x)=1 & \\
\bigvee c(\widehat{\chi(X)})(a)=c(\widehat{\chi(X)})(a)=c\left(\eta_{(A, \delta)}(X)\right)(a) . &
\end{aligned}
$$

Now, let $d$ be another closure operator on $P(A, \delta)$, such that $\eta_{(A, \delta)}:(P(A, \delta), d) \rightarrow$ $(F(A, \delta), c)$ is continuous. Then we have

$$
\begin{aligned}
\eta_{(A, \delta)}(d(X))(a)= & \chi \widehat{(d(X)})(a)=\bigvee_{x \in A} \chi(d(X))(x) \otimes \delta(a, x)= \\
& \bigvee_{x \in d(X)} \delta(a, x) \leq c\left(\eta_{(A, \delta)}(X)\right)(a)=c(\widehat{\chi(X)})(a) .
\end{aligned}
$$

Now, let $a \in d(X)$, then we have $1=\bigvee_{x \in d(X)} \delta(a, x) \leq c(\widehat{\chi(X)})(a)$, and it follows that $a \in \operatorname{core} c(\widehat{\chi(X)})=\varphi_{F(A, \delta), A}(c)(X)$. Hence, $d \leq \varphi_{F(A, \delta), A}(c)$.

Proposition 5.3. The categories $\operatorname{Set}(Q)[F]$ and $\operatorname{Set}(Q)[C]$ are isomorphic.

P r o of. The proof can be done analogously as the proof of Proposition 4.6. In fact, we define a functor $K: \operatorname{Set}(Q)[F] \rightarrow \operatorname{Set}(Q)[C]$, such that for any morphism $f:((A, \delta), c) \rightarrow((B, \gamma), d)$ in the category $\operatorname{Set}(Q)[F]$, we put

$$
K((A, \delta), c)=\left((A, \delta), \varphi_{F(A, \delta), C(A, \delta)}(c)\right), \quad J(f)=f .
$$

Then according to [11, Theorem 3.2 and Proposition 4.4],

$$
f:\left((A, \delta), \varphi_{F(A, \delta), C(A, \delta)}(c)\right) \rightarrow\left((B, \gamma), \varphi_{F(B, \gamma), C(B, \gamma)}(d)\right)
$$

is a morphism in $\operatorname{Set}(Q)[C]$ and the definition of $K$ is correct. Analogously, it can be showed that there exists the inverse functor $M$ such that for any morphism $g:((X, \rho), u) \rightarrow((Y, \tau), v)$ from the category $\operatorname{Set}(Q)[C]$,

$$
M((X, \rho), u)=\left((X, \rho), \varphi_{C(X, \rho), F(X, \rho)}(u)\right), \quad I(g)=g .
$$

Proposition 5.4. There exists a functor

$$
K: \operatorname{Set}(Q)[P]_{1} \rightarrow \operatorname{Set}(Q)[F] .
$$


Pro of. Let $f:((A, \delta), c) \rightarrow((B, \gamma), d)$ be a morphism in $\operatorname{Set}(Q)[P]_{1}$, then we set

$$
K((A, \delta), c)=\left((A, \delta), \varphi_{A, F(A, \delta)}(c)\right), \quad K(f)=f_{F} .
$$

From Theorem 2.2 and Proposition 2.2, it then follows that $K$ is a functor.

Analogously, as for the categories $\operatorname{Set}[\mathbf{P}]$ and $\operatorname{Set}[Z]$, the category $\operatorname{Set}(Q)$ can be considered to be a subcategory of $\operatorname{Set}(Q)[P]_{1}$ and also $\operatorname{Set}(Q)[F]$. In fact, we can introduce embedding functors

$$
1_{P}: \operatorname{Set}(Q) \rightarrow \operatorname{Set}(Q)[P]_{1}, \quad 1_{F}: \operatorname{Set}(Q) \rightarrow \operatorname{Set}(Q)[F],
$$

such that for any $(A, \delta) \in \operatorname{Set}(Q)$ and any morphism $f:(A, \delta) \rightarrow(B, \gamma)$ in $\operatorname{Set}(Q), 1_{P}(A, \delta)=\left((A, \delta), 1_{P(A, \delta)}\right), 1_{P}(f)=f$, and, analogously, $1_{F}(A, \delta)=$ $\left((A, \delta), 1_{F(A, \delta)}\right), 1_{F}(f)=f$, where $1_{P(A, \delta)}$ and $1_{F(A, \delta)}$ are considered to be (trivial) closure operators. It is cleat that $1_{P(A, \delta)} \in \widetilde{\mathcal{U}}(A)$ and $1_{P}(A, \delta) \in \operatorname{Set}(Q)[P]_{1}$. Then the power objects functors $P$ and $F$ can be extended to the power objects functors $P_{c l}, F_{c l}$ defined over categories $\operatorname{Set}(Q)[P]_{1}$ and $\operatorname{Set}(Q)[F]$, respectively, with values in the category $C S L A T_{c l}$ as follows.

$$
\begin{aligned}
& P_{c l}: \operatorname{Set}(Q)[P]_{1} \rightarrow C S L A T_{c l}, \quad F_{c l}: \operatorname{Set}(Q)[F] \rightarrow C S L A T_{c l} \text {, } \\
& ((A, \delta), c) \in \operatorname{Set}(Q)[P]_{1}, \quad P_{c l}((A, \delta), c)=(P(A, \delta), \subseteq, c), \quad P_{c l}(f)=f_{P}, \\
& ((A, \delta), d) \in \operatorname{Set}(Q)[F], \quad F_{c l}((A, \delta), d)=(F(A, \delta), \leq, d), \quad F_{c l}(f)=f_{F} .
\end{aligned}
$$

All these functors can be represented by the following commutative diagram.

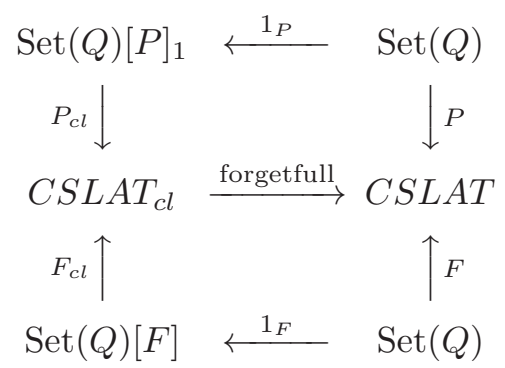

Proposition 5.5. There exists a natural transformation

$$
\eta: P_{c l} \rightarrow F_{c l} \circ K .
$$

Proof. Let $((A, \delta), c) \in \operatorname{Set}(Q)[P]$. Then we set $\eta_{((A, \delta), c)}(X)=\widehat{\chi X}$, for any $X \in P(A, \delta)$. According to Proposition 5.1,

$$
\eta_{((A, \delta), c)}: P((A, \delta), c) \rightarrow\left(F(A, \delta), \varphi_{A, F(A, \delta)}(c)\right)
$$

is continuous and it is also clear that it preserves $\bigvee$. Since $f_{F}\left(\widehat{\chi}_{X}\right)=\widehat{\chi}_{f(X)}$, it can be proved simply that $\eta$ is a natural transformation. 


\title{
CLOSURE THEORIES OF POWERSET THEORIES
}

\author{
REFERENCES
}

[1] EKLUnd, P.—GALÁn, M. A.—MEDinA, J.—OJEDA-ACIEGO, M.-VALVERDE, A.: Powerset of terms and composite monads, Fuzzy Sets Syst. 158 (2007), 2552-2574.

[2] EKLUnd, P.-GALÁn, M. A.-MEDinA, J.-OJEDA-ACIEGO, M.-VALVERDE, A.: Similarities between powersets of terms, Fuzzy Sets Syst. 144 (2004), 213-225.

[3] HÖHLE, U.: Many Valued Topology and its Applications. Kluwer Acad. Publ., Dordrecht, 2001.

[4] HÖHLE, U.: Fuzzy sets and sheaves. Part I, Basic concepts, Fuzzy Sets Syst. 158 (2007), $1143-1174$.

[5] HERRLICH, H.-STRECKER, C. G.: Category Theory, in: Sigma Ser. Pure Math. Vol. 1, Heldermann, Berlin, 1979.

[6] DE MITRI, C.—GUIDO, C.: Some remarks on fuzzy powerset operators, Fuzzy Sets Syst. 126 (2002), 241-251.

[7] ROSEnthal, K. I.: Quantales and Their Applications, in: Pitman Res. Notes Math. Ser., Vol. 234, Longman, Burnt Mill, Harlow, 1990.

[8] MOČKOŘ, J.: Morphisms in categories of sets with similarity relations, in: Proc. of IFSA Congress/EUSFLAT Conference (J. P. Carvalho et all., eds.), Lisabon, Portugal, 2009, pp. $560-568$.

[9] MOČKǑ̌, J.: Cut systems in sets with similarity relations, Fuzzy Sets Syst. 161 (2010), 3127-3140.

[10] MOČKOŘ, J.: Fuzzy sets and cut systems in a category of sets with similarity relations, Soft Comput. 16 (2012), 101-107.

[11] MOČKOட̌, J.: Extension principle for closure operators on fuzzy sets and cuts, Fuzzy Sets Syst. (to appear).

[12] MOČKǑ̌, J.: Powerset Operators of Fuzzy Objects, (to appear).

[13] PREUSS, G.: Foundations of Topology: An Approach to Convenient Topology. Kluwer Acad. Publ., Dordrecht, 2002.

[14] RODABAUGH, S. E.: Powerset operator foundation for poslat fuzzy sst theories and topologies, in : Mathematics of Fuzzy Sets: Logic, Topology and Measure Theory (U. Höhle et all., eds.), The Hnadbook of Fuzzy Sets Series, Vol. 3, Kluwer Acad. Publ., Dordrecht, 1999, pp. 91-116.

[15] RODABAUGH, S. E.: Powerset operator based foundation for point-set lattice theoretic (poslat) fuzzy set theories and topologies, Quaest. Math. 20 (1997), 463-530.

[16] RODABAUGH, S. E.: Relationship of algebraic theories to powerset theories and fuzzy topological theories for lattice-valued mathematics, Int. J. Math. Math. Sci. 2007 (2007), $1-71$.

[17] RODABAUGH, S. E.: Relationship of algebraic theories to powersets over objects in Set and SetxC, Fuzzy Sets Syst. 161 (2010), 453-470.

[18] SOLOVYOV, S. A.: Powerset oeprator foundations for catalg fuzzy set theories, Iran. J. Fuzzy Syst. 8 (2001), 1-46.

[19] MANES, E. G.: Algebraic Theories. Springer-Verlag, Berlin, 1976.

[20] MANES, E. G.: A class of fuzzy theories, J. Math. Anal. Appl. 85 (1982), 409-451.

[21] NOVÁK, V.-PERFILIJEVA, I.-MOČKOř, J.: Mathematical Principles of Fuzzy Logic. Kluwer Acad. Publ., Dordrecht, 1999.

[22] GERLA, G.-SCARPATI, L.: Extension principles for fuzzy set theory, J. Infor. Sci. 106 (1998), 49-69.

[23] NGUYEN, H. T.: A note on the extension principle for fuzzy sets, J. Math. Anal. Appl. 64 (1978), 369-380. 


\title{
JIŘí MOČKOř
}

[24] HE, Q.-LI, H.-CHEN, C. L. P.-LEE, E. S.: Extension principles and fuzzy set categories, Comput. Math. Appl. 39 (2000), 45-53.

[25] YAGER, R. R.: A characterization of the extension principle, Fuzzy Sets Syst. 18 (1996), 205-217.

[26] ZENG, W.-ZHAO, Y.-LI, H.: Extension principle of interval-valued fuzzy set, in: Proc. of the 2nd Internat. Conf. of Fuzzy Inform. and Eng.-ICFIE '07 (Cao, Bing-Yuan, ed.), Guangzhou, China, 2007, Adv. Soft Comput. 40 (2007), Springer, Berlin, 2007, pp. $125-137$.

[27] WYLER, O.: Fuzzy logic and categories of fuzzy sets, in: Non-Classical Logics and Their Applications to Fuzzy Subsets, Theory Decis. Libr., Ser. B. Vol. 32, Kluwer Acad. Publ. Dordrecht, 1995, pp. 235-268.

[28] ZADEH, L. A.: Fuzzy sets, Inform. Control 8 (1965), 338-353.

[29] ZHAI, JIAN-YIN: General extension principle, Fuzzy Syst. Math. 9 (1995).

Received November 21, 2015

\author{
University of Ostrava \\ Institute for Research and \\ Applications of Fuzzy Modeling \\ Ostrava 1 \\ CZECH REPUBLIC \\ E-mail: jiri.mockor@osu.cz
}

